Acta bot. bras. I(2):103-119 (1988) supl.

\title{
ANÁLISE DA ESTRUTURA DA COMUNIDADE DE DIATOMÁCEAS (BACILLARIOPHYCEAE) EM DUAS ESTAÇÕES DO SISTEMA GUAIBBA, RIO GRANDE DO SUL, BRASH.
}

\author{
Eduardo A. Lobo (1) \\ Lezilda Carvalho Torgan $(1,2)$
}

\begin{abstract}
RESUMO - Através da análise da composição específica e da diversidade da comunidade de diatomáceas, em duas estações do sistema Guaíba (RS, Brasil), nos meses de setembro e novembro de 1975, foi encontrada uma diferença significativa na estrutura dessa comunidade. Essa diferença deve-se, provavelmente, à presença exclusiva de Achnanthes inflata (Kütz.) Grun. var. elata (Leud. Fort.)Hust., A. lanceolata (Bréb.)Grun. var. lanceolata, Cocconeis placentula Ehr., Cyclotella meneghiniana Kütz., Cymbella minuta Hilse ex Rabh., Aulacosira distans (Ehr.) Sim., Navicula mutica Kütz., Surirella robusta Ehr. var. splendida (Ehr.) Van Heurck, Synedra ulna (Nitz.)Ehr., espécies abundantes, que refletem características ambientais distintas, pelo fato de apresentarem uma diferenciaçāo em suas tolerâncias ecológicas. Foram identificados um total de 114 táxons, 57 a níveis específicos e infra-específicos, dentre os quais 14 constituem-se em primeira referência para o Guafba e quatro são citaçōes novas para o Estado.
\end{abstract}

\begin{abstract}
Through the analysis of specific composition and diversity of diatom community, in two sampling areas of the Guaiba system (RS, Brasil) in September and October 1975, a meaningful difference in the structure of that community was found. Such difference is probably due to exclusive presence of Achnanthes inflata (Kütz.)Grun. var. elata (Leud. - Fort.)Hust., A. lanceolata(Bréb.)Grun. var. lanceolata, Cocconeis placentula Ehr., Cyclotella meneghiniana Kütz., Cymbella minuta Hilse ex Rabh., and of Aulacosira distans(Ehr.) Sim., Navicula mutica Kütz., Surirella robusta Ehr. var. splendida (Ehr.)Van Heurck, Synedra ulna(Nitz.)Ehr., abundant species which reflect different environmental characteristics because of their different ecological tolerances. A total of 114 taxa have been identified; 57 of them to specific and infraespecific levels, 14 of which mentioned for the first time in the Guaiba system and $\mathbf{4}$ for the first time in the state.

Key-words: Algae, Bacillariophyceae, structure of vommunity.
\end{abstract}

\section{Introdução}

Os sistemas lóticos caracterizam-se por apresentar condiçōes ambientais que estão variando intensamente no espaço e no tempo (Odum 1972, Margalef 1974). Essa variação ambiental sugere que as comunidades, que ocorrem nesses sistemas, devem ajustar-se, modificando sua estrutura, através do número de indivíduos das espécies presentes e/ou da substituição de algumas espécies por outras, de acordo com os intervalos de tolerância que apresentam para determinados fatores ambientais.

Em geral, os trabalhos de limnologia em sistemas Ióticos do Estado do Rio Grande do Sul e, em particular, os referentes ao sistema Guaîba, são escassos. A maioria é de caráter descritivo e taxonômico.

(1) Museu de Ciências Naturais da Fundação Zoobotânica do Rio Grande do Sul. Caixa Postal 1188, 90610, Porto Alegre, RS, Brasil.

(2) Bolsista do CNPq. 
Com relação a estudos de taxonomia e ecologia da ficoflórula do Guaiba, o Departamento Municipal de Águas e Esgotos de Porto Alegre (DMAE) realizou um levantamento de características físicas, químicas e biológicas, a fim de avaliar a qualidade de suas águas (Porto Alegre - DMAE 1971). Os resultados são apresentados sob a forma de gráficos e tabelas, sendo que, para o fitoplâncton, foi feita identificação a nível genérico e contagens do número de organismos por litro. Togan e Aguiar (1974) efetuaram um levantamento taxonômico das espécies de diatomáceas que ocorreram no inverno de 1972, estabelecendo uma relação das espécies mais frequentes com as condições de saprobidade, através do enquadramento destas em sistemas de sapróbios citados em literatura. Posteriormente o DMAE verificou a ocorência de superpopulaçōes de determinados gêneros de organismos planctônicos nos verōes de 1971, 1972 e 1973 (Porto Alegre - DMAE 1974). Togan e Aguiar (1978) realizaram um estudo taxonômico de 159 táxons especificos e infra-especificos de diatomáceas em 6 pontos do Guaî̉a, levando em consideração sua distribuição, frequência relativa, bem como sua variação em relação às estaçōes de coleta no inverno de 1972 e verão de 1973. Num histórico de monitoramento realizado pelo DMAE no Guaiba, no período de 1970 a 1978 (Porto Alegre - DMAE 1978), foi reformulado o plano de amostragem, a fim de obter uma melhor caracterização da área, através de análises físicas, químicas e biológicas, onde é apresentado uma lista de 167 espécies de diatomáceas, amostradas no período de 1977-78, referindo as espécies exclusivas para cada estação de coleta. Seguindo, o DMAE estabeleceu a correlação entre o fitoplâncton e clorofila "a", bem como a relação entre a biomassa com sua produtividade e teores de nutrientes, mencionando as espécies de algas que predominam nos "blooms" de verão (Porto Alegre - DMAE 1981). Torgan (1984) apresentou os resultados do levantamento de 61 táxons de diatomáceas, efetuado no ano de 1975 em 5 estações de coleta, situadas no canal e praias à margem esquerda do Guaiba, citanido os mais representativos pela sua abundância.

O conhecimento da flórula algológica do Guaíba e sua ecologia é, portanto, bastante restrito, especialmente no que se refere ao estudo das estruturas fitoplanctônicas. Assim, o presente trabalho tem por objetivo caracterizar a estrutura da comunidade de diatomáceas, por ser o grupo mais representativo do fitoplâncton do sistema Guaiba, em uma secção deste, e estabelecer suas relaçōes com a variabilidade do ambiente físico e químico.

Para cumprir com o objetivo geral, foram formulados os seguintes objetivos especificos: identificação das espécies de diatomáceas ocorrentes em uma secção das águas do Guaiba; estabelecimento da estrutura da comunidade, incluíndo densidade celular, riqueza de espécies, diversidade especifica, uniformidade das espécies, similaridade na composição especifica, espécies abundantes; e determinação da relação entre a estrutura da comunidade e a variabilidade das características do ambiente físico e químico.

\section{Material e Métodos}

Foram selecionadas duas estaçōes de amostragem no sistema Guaîba, para análise biológica, denominadas 1 e 2 , situadas eqüidistantes do ponto a $30^{\circ} 08^{\prime} 02^{\prime \prime} \mathrm{S}$ e $51^{\circ} 17^{\prime} 58^{\prime \prime} \mathrm{W}$, pelo fato delas corresponderem às estaçōes de coleta, destinadas para análises físicas e químicas, 51-b (Lançamento do efluente da ex-Indústria de Celulose Borregaard, próxima ao canal), e 50 (frente ao Morro da Ponta Grossa, no canal), fixadas pelo Departamento Municipal de Águas e Esgotos de Porto Alegre (Fig. 1). As coletas foram realizadas juntamente com os técnicos desse Departamento, nos meses de setembro e novembro de 1975.

Foram coletadas amostras de 1 litro obtidas com garrafa de Kemmerer, no sentido horizontal, logo abaixo da superficie da água, sendo essas fixadas com formalina a $4 \%$. 
Analisou-se, para cada estação, uma sub-amostra de $10 \mathrm{ml}$, que foi preparada segundo a técnica de Müller-Melchers e Ferrando (1956). O material foi distribuído em 3 lâminas permanentes para identificação e contagem. As amostras observadas encontram-se depositadas no Herbário Prof. Dr. Alarich Schultz, no Museu de Ciências Naturais da Fundação Zoobotânica do Rio Grande do Sul, sob os números HAS 6610(Lâminas 1032-4); 6611(Lâminas 1029-31); 6615(Lâminas 1107-9); 6616(Lâminas 1110-2).

Para a análise qualitativa e quantitativa das diatomáceas, foi utilizado um microscópio de pesquisa Leitz, modelo Dialux. Empregou-se o sistema de Simonsen(1979) para o enquadramento taxonômico das espécies e para a identificação destas foram utilizadas as seguintes obras: Cleve-Euler(1932, 1951-5); Compère(1982); Emiliani(1973); Frenguelli(1942); Hendey(1964); Huber-Pestalozzi(1942); Hustedt(1930); Patrick e Reimer(1966, 1975); Peragallo(1897-1908); Stoermer(1963) e Van Heurck(1880-1). Os dados físicos e químicos foram fornecidos pelo Centro de Estudos de Saneamento Básico-CESB do DMAE, através do relatório interno Porto Alegre-DMAE(1976).

Para determinação da estrutura da comunidade, foram utilizados os seguintes indicadores: densidade celular expressa em cél/l; riqueza de espécies expressa em termos do número total de espécies presentes; diversidade especffica utilizando o índice de diversidade de Shannon e Weaver(1949, apud Odum 1972) uniformidade das espécies através do indice de uniformidade de Pielou(1975); similaridade na composiçāo especffica utilizando o coeficiente de similaridade de Jaccard (Estrada et al. 1975); e espécies abundantes conforme os critérios de Lobo(1984).

Foi utilizado o teste " $t$ " de significância, para comparar as diversidades específicas entre ambas estaçōes de amostragem, nos respectivos meses de coleta. Trabalhou-se com intervalo de confiança de $95 \%$.

\section{Resultados e Discussão}

1. Composição taxonômica:

A análise qualitativa das amostras coletadas, nas estaçōes 1 e 2, em setembro e novembro de 1975, no sistema Guaîba, revelou a presença de 114 táxons de diatomáceas, 14 dos quais se constituem na primeira citação para o Guaíba. Os taxa identificados à níveis genérico, éspecífico e infra-específico encontram-se na seguintes relação:

\section{CENTRALES}

\section{THALASSIOSIRACEAE}

*Aulacosira ambigua (Grunow) Simonsen var. ambigua; *A. distans (Ehrenberg) Simonsen; A. granulata (Ehrenberg) Simonsen var. granulata; A. granulata (Ehrenberg) Simonsen var. angustissima (O. Müller) Simonsen; ${ }^{*}$. granulata (Ehrenberg) Simonsen var. valida (Hustedt) Simonsen; *A. pseudogranulata (Cleve-Euler) Simonsen; Aulacosira sp. Cyclotella meneghiniana Kützing; $C$. stelligera (P.T. Cleve et Grunow) Van Heurck; $C$. striata (Kützing) Grunow

Thalassiosira spp.

MELOSIRACEAE

Paralia sulcata (Ehrenberg) P.T. Cleve

COSCINODISCACEAE

Coscinodiscus spp.

HEMIDISCACEAE

Actinocyclus sp.

RHIZOSOLENIACEAE

Rhizosolenia calcaravis Schultze 


\section{EUPODISCACEAE}

Pleurosira laevis (Ehrenberg) Compère

\section{PENNALES}

\section{DIATOMACEAE}

Asterionella japonica P.T. Cleve

Opephora sp.

Synedra goulardi Brébisson var. goulardi; ${ }^{*}$ S. rumpens Kützing var. familiaris (Kützing) Hustedt; *S. rumpens Kützing var. fragilarioides Grunow; $S$. ulna (Nitzsch) Ehrenberg EUNOTIACEAE

Eunotia flexuosa Brébisson ex Kützing var. flexuosa; E. lunaris (Ehrenberg) Grunow; $E$. praerupta Ehrenberg var. bidens (Ehrenberg) Grunow; *E. revoluta Cleve-Euler; Eunotia sp.

\section{ACHNANTHACEAE}

Achnanthes exigua Grunow var. constricta (Grunow) Hustedt; $A$. inflata (Kützing) Grunow var. inflata; ${ }^{*} A$. inflata (Kützing) Grunow var. elata (Leuduger-Fortmorel) Hustedt; $A$. lanceolata (Brébisson) Grunow var. lanceolata; A. lanceolata (Brébisson) Grunow var. dubia Grunow; Achnantes spp.

Cocconeis placentula Ehrenberg; Cocconeis spp.

NAVICULACEAE

Amphipleura pellucida Kützing

Cymbella affinis Kützing var. affinis; C. minuta Hilse ex Rabenhorst; C. tumida (Brébisson ex Kützing) Van Heurck var. tumida; Cymbella sp.

Diploneis elliptica (Kützing) P.T. Cleve var. elliptica; Diploneis spp.

${ }^{\star}$ Frustulia rhomboides (Ehrenberg) De Toni var. capitata (A. Mayer)Patrick; Frustulia sp.

Gomphonema parvulum Kützing; G. truncatum Ehrenberg var. truncatum; Gomphonema spp.

Gyrosigma scalproides (Rabenhorst) P.T. Cleve var. scalproides; Gyrosima sp.

Navicula capitata Ehrenberg var. hungarica (Grunow) Ross; *N. lateropunctata Wallace; $N$. mutica Kützing; $N$. pupula Kützing var. pupula; ${ }^{*} N$. rhyncocephala Kützing var. germainii (Wallace) Patrick; Navicula spp.

${ }^{*}$ Neidium affine (Ehrenberg) Pfitzer var. longiceps (Gregory)P.T. Cleve; ${ }^{*} N$ : hankensis Skvortzow

Pinnularia acrosphaeria Wm. Smith var. acrosphaeria; P. aperta Frenguelli var. doellojuradoi Frenguelli

$P$. borealis Ehrenberg var. borealis; ${ }^{*} P$. borealis Ehrenberg var. rectangularis Carlson; $P$. braunii (Grunow) P.T. Cleve var. amphicephala (A.Mayer) Hustedt; $P$. divergens Wm. Smith; P. mesolepta (Ehrenberg) Wm. Smith var. mesolepta; Pinnularia spp.

EPITHEMIACEAE

*Rhopladodia musculus (Kützing) O. Müller var. musculus

NITZSCHIACEAE

Hantzschia amphioxys (Ehrenberg) Grunow

Nitzschia acicularis Wm. Smith; $N$. brevissima Grunow; $N$. palea (Kützing) Wm. Smith; $N$. tryblionella Hantzsch var. victoriae Grunow; Nitzschia sp.

SURIRELLACEAE

Surirella robusta Ehrenberg var. splendida (Ehrenberg) Van Heurck

Surirella spp.

*Primeira citação para o Guaíba. 
Os táxons estão distribuídos em 13 famnlias e 27 gêneros. A famnia Naviculaceae e os gêneros Navicula Bory e Pinnularia Ehr. foram os melhores representados em número de espécies, variedades e formas taxonômicas. Dentre as espécies identificadas, são citações novas para o Estado as de Eunotia revoluta Cleve-Euler, Navicula lateropunctata Wallace, Navicula rhyncocephala Kütz. var. germainii (Wallace) Patrick e P. borealis Ehr. var. rectangularis Carlson.

É importante mencionar que Asterionella japonica Cleve, Paralia sulcata (Ehr.) P.T. Cleve (=Melosira sulcata(Ehr.) Kütz.) e Rhizosolenia calcaravis Schultze, espécies tipicamente marinhas, comuns de ocorrer nas praias da costa do Rio Grande do Sul (Corte-Real \& Callegaro 1973), foram encontradas isoladas, sem cromoplastos ou em fragmentos nas amostras analisadas das estações 1 e 2, do mês de novembro. Esse fato indica que, provavelmente, essas espécies tenham se fixado aos cascos de embarcações e transportadas no sentido S-N, ou ainda trazidas pela ocorrência da inversão do fluxo de escoamento do Guaíba que, segundo Ferreira e Dewes (1984), em período de estiagem prolongada, é aparentemente proporcional ao ciclo da maré alta e à ação do vento no quadrante sul. As referidas espécies são consideradas, portanto, formas acidentais relativas ao local de estudo.

Por outro lado, foi observada a presença de representantes dos gêneros Actinocyclus, Coscinodiscus e Thalassiosira, bem como das espécies Pleurosira laevis(Ehr.)Compère (=Siddulphia laevis Ehr. e Cyclotella striata(Kütz.)Grun. Essas espécies são mencionadas na literatura como típicas de águas salobras, no entanto, já foram registradas para o Guaiba e seus afluentes (Porto Alegre-DMAE 1978, 1981, Martau et al. 1977). Em nosso material, esses táxons apresentam-se sem cromoplastos e, dado que os autores já citados não observaram essa característica ou trabalharam com material oxidado, futuras investigações nessa área permitirão definir se essas espécies pertencem ou não à comunidade de diatomáceas do sistema Guaíba. Por esse motivo, estas não foram consideradas na análise da estrutura da comunidade.

\section{2. Índices de diversidade:}

A figura 2 apresenta a variação dos índices de diversidade da comunidade de diatomáceas nas estações 1 e 2, nos meses de setembro e novembro de 1975. Se compararmos as estações de amostragem, nos respectivos meses de coleta, observa-se que, no mês de setembro não houve diferenças significativas na diversidade especifica, porém, o número de indivíduos foi maior na estação 1 do que na estação 2 .

A figura 3 apresenta as espécies abundantes para ambas estaçōes no mês de setembro. Observa-se que, na estação 1 , houve um número maior de espécies do que na estação 2, sendo Aulacosira granulata (Ehr.) Simonsen var. granulata o táxon que atingiu a máxima abundância (24\%). Esta espécie apresentou, também, a máxima abundância na estação 2 (11\%), sendo o único táxon que ocorreu em ambas estações. A baixa similaridade observada entre as espécies abundantes de ambas estações $(8,3 \%)$, segundo $o$ índice de Jaccard, sugere que há uma diferenciação dos requerimentos ambientais para essas espécies; as quais, segundo Saiz (1980), formam o núcleo central de uma comunidade, sendo a base da estrutura desta. Com base nesse critério, é provável que a dissimilaridade observada entre as espécies abundantes e, portanto, a diferenciação de requerimentos ambientais seja um dos principais fatores que determina a estrutura das comunidades comparadas.

$\mathrm{Na}$ figura 2 observa-se que, no mês de novembro, a estação 1 apresentou uma diversidade específica significativamente maior do que a estação 2 . Porém, se compararmos os valores totais obtidos para esses indicadores em ambos os meses, vemos que foram menores aos obtidos no mês de setembro. Esses resultados indicam a ocorrência de uma 
diferenciação temporal e, provavelmente, um ajuste de espécies com tolerâncias ecológicas diferentes. Verificou-se essa diferença com baixos índices de similaridade obtidos, $20,3 \%$ para a estação 1 entre os meses de setembro e novembro e $18,8 \%$ para a estação 2, nesses meses. Em novembro, houve um número maior de espécies na estação 1 do que na estação 2. No entanto, o número de indivíduos foi menor, mas, pelo fato de termos maior uniformidade na distribuição destes, observou-se um alto valor para a diversidade especifica.

A figura 4 apresenta as espécies abundantes para ambas estações no mês de novembro. Observa-se que, na estação 1, houve um número maior de espécies, sendo Aulacosira granulata var. granulata o táxon de máxima abundância. Considerando a similaridade entre as espécies abundantes de ambas estações, constatou-se que esta foi de $71,4 \%$, significativamente maior do que a observada no mês de setembro, sendo $A$. granulata var. granulata, A. distans (Ehr.) Simonsen, A. ambigua (Grun.) Simonsen var. ambigua, A. pseudogranulata (Cleve-Euler)Simonsen e Aulacosira sp., os táxons comuns. Pode-se observar, ainda, que esses constituem o número total de táxons abundantes encontrados na estação 2. Esses resultados indicam que a diferença na estrutura das comunidades devese, provavelmente, à presença exclusiva das espécies abundantes Cocconeis placentula Ehr. e Cyclotella meneghiniana Kütz. na estação 1, as quais, conforme Krebs (1972), apresentam uma correspondência significativa entre suas tolerâncias ecológicas e as condições ambientais locais estabelecendo, portanto, uma comunidade com uma estrutura diferente.

\section{Análises físicas e químicas:}

Através dos resultados das análises físicas e químicas, é possível eatabelecer diferenças de ordem entre as estaçōes 1 e 2 durante os meses de setembro e novembro, levando-se em consideração que os dados fornecidos são valores discretos, referentes a uma amostra mensal, o que impossibilita seu tratamento estatístico.

Com base nessa observação, a figura 5 mostra que houve no mês de setembro, diferença para a concentração total de sólidos decantáveis, com seu máximo na estação 1. A temperatura manteve-se estável, se considerarmos que a diferença entre ambas estações foi de $0,5^{\circ} \mathrm{C}$. O conteúdo de oxigênio dissolvido foi maior na estação 1 sendo que, para a mesma estação, a demanda bioquímica de oxigênio, após 5 dias, atingiu valores de $2 \mathrm{mg} / \mathrm{l}$, com uma diferença de $1,6 \mathrm{mg} / \mathrm{l}$, com relação à estação 2 .

$\mathrm{Na}$ figura 6, observa-se que no mês de setembro a concentração de nutrientes foi maior na estação 2 que na 1, com excessão do fosfato. Os compostos inorgânicos dissolvidos apresentam concentraçōes maiores na estação 1 que na 2, com exceção do magnésio, sendo que, a diferença máxima foi verificada para o cálcio, com um valor de 1,6 $\mathrm{mg} / \mathrm{l}$.

Esses resultados indicam que, no mês de setembro, a estação 1 caracterizou-se por apresentar alta concentração de sólidos decantáveis e de compostos inorgânicos dissolvidos, elevado conteúdo de oxigênio, alta demanda bioquímica de oxigênio, mas a concentração de nutrientes foi baixa quando comparada com os valores obtidos para a estação 2 .

$\mathrm{Na}$ figura 5, observa-se que, no mês de novembro, a concentração de sólidos decantáveis atingiu seu máximo valor na estação 1, maior que o observado no mês de setembro, assim, em ambos meses a estação 1 caracterizou-se por apresentar as mais altas concentraçōes. A temperatura pode, assim como no mês de setembro, ser considerada estável, dado que a diferença entre ambas estações foi de $0,5^{\circ} \mathrm{C}$. $\mathrm{O}$ conteúdo de oxigênio dissolvido manteve-se constante, no entanto, a demanda bioquímica de oxigênio, após 5 dias, apresentou uma diferança de 0,2 mg/l, com seu máximo na estação 2 . 
Na figura 6, observa-se que, no mês de novembro, houve uma concentração de nutrientes maior na estação 1 que na estação 2 , sendo que a máxima diferença foi verificada para o sulfato, com uma concentração de $1,3 \mathrm{mg} /$. Os compostos inorgânicos dissolvidos, sódio e potássio, não mostram diferenças entre ambas estações, ao contrário do que aconteceu no mês de setembro, onde as maiores concentraçōes foram registradas para a estação 1.

Esses resultados indicam que, no mês de novembro, a estação 1 caracterizou-se por apresentar alta concentração de sólidos decantáveis e de nutrientes, no entanto, a demanda bioquímica de oxigênio, após 5 dias, foi baixa, quando comparada com os valores obtidos para a estação 2 .

\section{Relação comunidade - ambiente:}

Os resultados das análises dos índices de diversidade indicam que no mês de setembro, não houve diferenças significativas na diversidade especffica. Porém, considerando as diferenças na composição especiffica, foi possivel identificar dois tipos de estruturas. Essa diferença poderia ser explicada em termos da diferenciação de requerimentos ambientais das espécies abundantes que pertencem às respectivas comunidades.

A baixa similaridade observada entre as espécies abundantes das estaçōe 1 e 2 sugere que a diferença na estrutura das comunidades deve-se, provavelmente, à presença exclusiva de Achnanthes inflata (Kütz.)Grunow var. elata, A. lanceolata (Bréb.)Grün. var. lanceolata, Cymbella minuta (Hilse ex Rabh.) e Nitzschia palea (Kütz.)Wm.Smith na estação 1 e de Aulacosira distans, Cocconeis placentula, Navicula mutica Kütz., Surirella robusta Ehr. var. splendida (Ehr.) Van Heurck e Synedra ulna (Nitzsch)Ehr. na estação 2.

Os resultados das análises físicas e químicas indicam que, no mês de setembro, a estação 1 caracterizou-se por apresentar uma alta concentração de sólidos decantáveis e de compostos inorgânicos dissolvidos, alta demanda bioquímica de oxigênio e baixa concentração de nutrientes, condições que, segundo Billings e DeHaas (1971), Lange-Bertalot (1979) e Schoeman (1979), indicam níveis B - alfa-mesossapróbios de poluição. Essa classificação é apoiada pela tolerância à poluição das espécies Achnanthes lanceolata (Lange-Bertalot 1979) e, principalmente, Nitzschia palea (Kobayasi \& Mayama 1982), reconhecida como característica de águas excessivamente poluídas (Schroeder 1939, Van Landingham 1964, Schoeman 1976, 1977, Lange-Bertalot 1979). Descy (1979) estabeleceu que os compostos inorgânicos dissolvidos, principalmente cálcio e potássio, são fatores que aumentam a poluição. No entanto, espécies dos gêneros Achnanthes e Cymbella são capazes de tolerar altas concentraçōes (Werrer 1977).

A estação 2 caracterizou-se por apresentar uma baixa concentração de sólidos decantáveis e de compostos inorgânicos dissolvidos, baixa demanda bioquímica de oxigênio e uma alta concentração de nutrientes. Segundo Lange-Bertalot (1979) e Schoeman (1979), essas condiçōes indicam níveis B - mesossapróbios de poluição, sendo caracteristicas desse ambiente, moderadamente poluído, as espécies Aulacosira distans (Luchini \& Verona 1972, Lowe 1974), Cocconeis placentula (Luchini \& Verona 1972, Sládecek 1973, Lowe 1974, Lange-Bertalot 1979) e Synedra ulna (Luchini \& Verona 1972, Sládecek 1973, Lowe 1974). A concentração de nutrientes, principalmente de nitrogênio total, foi alta, o que, segundo Werner (1977), estabelece condições favoráveis para o desenvolvimento dessas espécies, dado que são tolerantes a altos teores de nitrogênio (1-3 mg/l). Porém, essas concentrações refletem níveis mesossapróbios de poluição (Werner 1977).

No mês de novembro, a estação 1 apresentou diversidade significativamente maior que a estação 2 e, portanto, uma diferença na estrutura das comunidades observadas. Neste caso, a diferença deve-se, provavelmente, à presença exclusiva das espécies abundantes Cocconeis placentula e Cyclotella meneghiniana na estação 1, dado que o total 
de espécies abundantes na estação 2, foi também abundante na estação 1. Assim, é de se esperar que os táxons acima referidos apresentem adaptaçōes especfficas às condiçōes ambientais locais, as quais favorecem seu desenvolvimento.

Os resultados das análises físicas e quimicas indicam que, no mês de novembro, a estação 1 caracterizou-se por apresentar alta concentração de sólidos decantáveis e de nutrientes e baixa demanda bioquímica de oxigênio. Segundo Billings e DeHaas (1971) e Descy (1979), as duas primeiras características indicam condiçōes de poluiçāo "moderada", as quais correspondem a niveis B - mesossapróbios. Segundo Luchini e Verona (1972), Sládecek (1973) e Lowe (1974), Cocconeis placentula e Cyclotella meneghiniana são espécies que toleram níveis oligo-mesossapróbios de poluição e, portanto, características do ambiente descrito.

De acordo com Van Landingham (1964), Billings e DeHaas (1971) e Wemer (1977), alta concentração de sólidos decantáveis produz turvamento d’água e, como consequeência, diminuição da camada trofogênica por efeito a uma menor penetração da luz. Em nosso caso, a estação 1 apresentou alta concentração de sólidos decantáveis. $O$ tempo da permanência prolongada desse material nas capas superficiais do corpo d’água, limita 0 processo da fotossíntese, por efeito da diminuição da penetração da luz. Portanto, as espécies que caracterizam as comunidades desse "habitat" deveriam apresentar condiçōes especfficas de flotabilidade para se manterem entre os limites da zona trofogênica e aproveitar, ao máximo, a disponibilidade de nutrientes. As diatomáceas de sistemas lóticos são, geralmente, bentônicas ou epiffiticas, sendo pouco comuns as espécies verdadeiramente planctônicas (Werner 1977). No entanto, Cyclotella meneghiniana é referida na literatura como euplanctônica (Lowe 1974), condição que favorece a manutenção dessa espécie nas capas superficiais d’água. Rice (1938 apud Van Landigham 1964) cita essa espécie como abundante nas capas sub-superficiais em sistemas lóticos. A disponibilidade de nutrientes, principalmente fosfato e sulfato, foi alta, condição que, provavelmente, favoreceu o desenvolvimento das espécies Cocconeis placentula e Cyclotella meneghiniana, pelo fato de serem reconhecidas como abundantes em ambientes com altos teores de nutrientes sob condiçōes mesotróficas (Luchini \& Verona 1972, Lowe 1974, Werner 1977).

A temperatura apresentou diferença de $0,5^{\circ} \mathrm{C}$ entre as estaçōes 1 e 2 , em ambos meses, com uma variação de 16,5 a $19^{\circ} \mathrm{C}$. Van Landingham (1964) estabeleceu que a máxima abundância das diatomáceas está correlacionada com a temperatura d’ăgua e os nutrientes dissolvidos. Porém, a pequena diferença na temperatura observada em ambas épocas e o intervalo registrado, sugerem que as espécies encontradas na comunidade sāo características de águas temperadas com tolerâncias para as temperaturas que correspondem às condiçōes ambientais locais (Patrick 1971).

A estação 1 apresentou, tanto em setembro como novembro, alta concentração de sólidos decantáveis, maior do que a registrada na estação 2 . Esses fato deve-se, provavelmente, à descarga de despejos industriais da ex-indústria de celulose Borregaard, cujo vertedouro situa-se próximo à estação de coleta, dado que os sólidos decantáveis constituem uma das mais importantes fontes de poluição de uma indústria de celulose (Billings \& DeHaas 1971), junto com compostos orgânicos solúveis, principalmente, organosulfurados e metanol (Fox 1977), estabelecendo, portanto, condiçōes desfavoráveis para o desenvolvimento das espécies (Kobayasi \& Mayama 1982). Segundo o sistema de Lange-Bertalot (1979), a estação 1 apresentou, no mês de setembro, níveis B-alfa-mesossapróbios ou criticamente poluído e, no mês de novembro, níveis B-mesossapróbios ou moderadamente poluído. Assim, os sólidos decantáveis provenientes da descarga de despejos industriais da ex-indústria de celulose Borregaard foram uma das principais fontes de poluição e fator importante na determinação da estrutura das comunidades. A diferença entre os niveis de poluição identificados em ambos os meses, pode ser constatada considerando a alta de- 
manda bioquímica de oxigênio registrada no mês de setembro, dado que esse fator avalia o efeito que têm os resíduos orgânicos de uma indústria de celulose em um corpo d'água, sendo, portanto, um bom indicador de poluição (Billings \& DeHaas 1971).

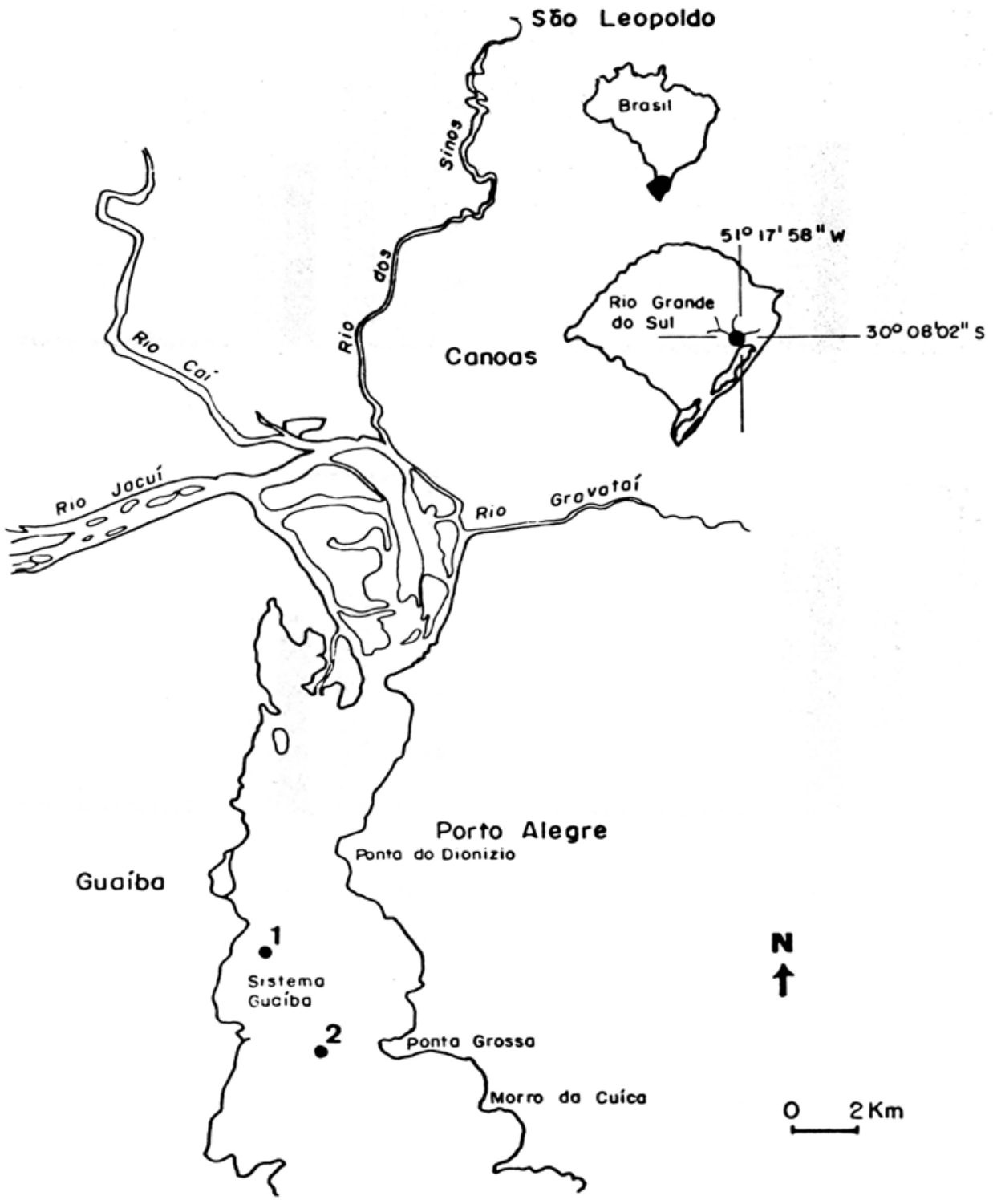

Fig. 1. Posiçăo geográfica do sistema Guaßba com as estaçőes de amostragem. 

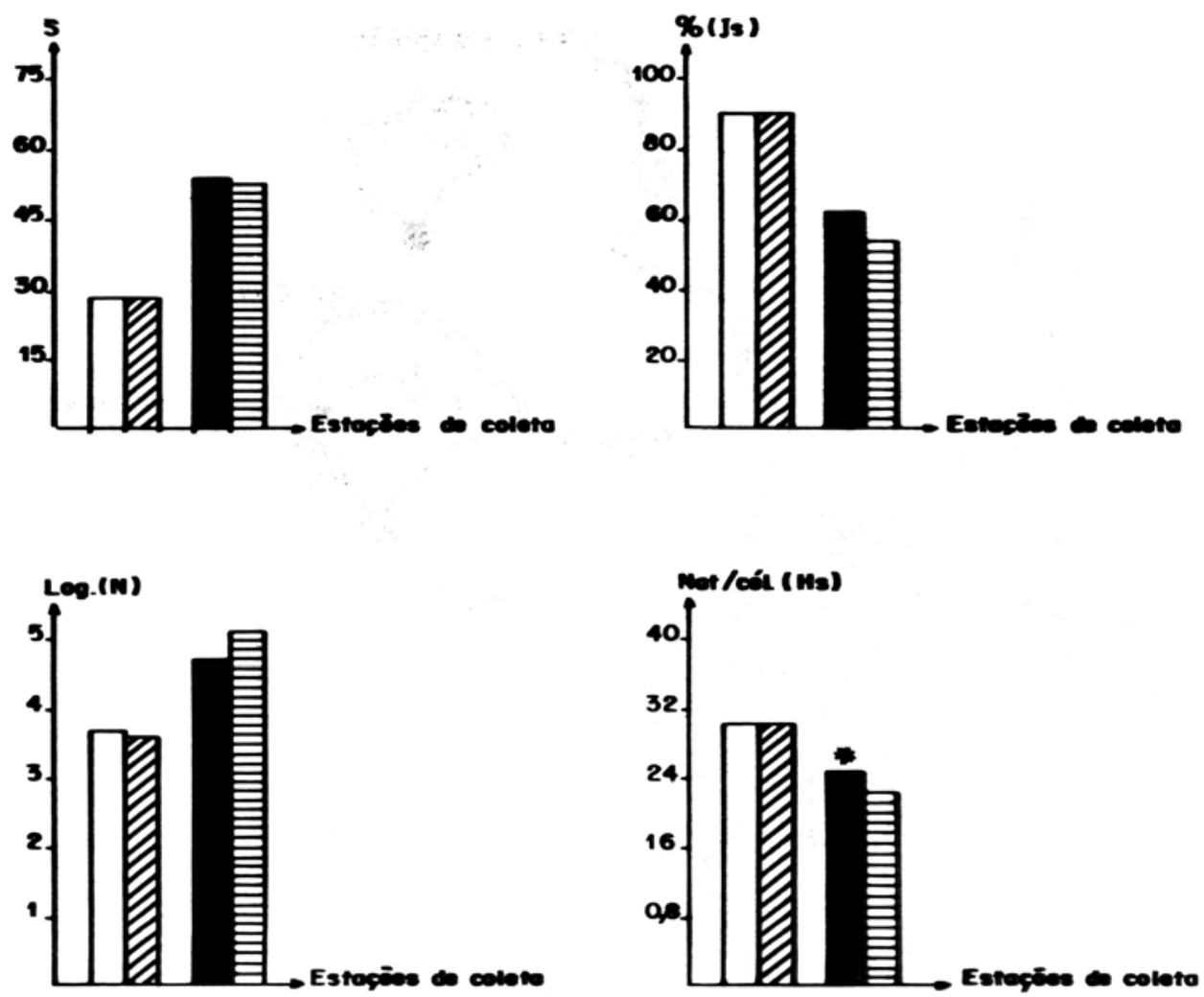

$\square$ Sot. 1975 Est. 1

Nov. 1975 Est. 1

Sot. 1975 Est.2

Nov. TeTs Est. 2

Fig. 2. Variaçōes dos Indices de diversidade (S: riqueza de espécies, Js: indice de unifornidade, Nk número total de células/itro, $\mathrm{Hs}$ indice de diversidade especifica), nas estaçōies de coleta 1 e 2, em um transecto do sisterna Guaiba, durante os meses de setembro e novernbro de 1975. (") diferença significativa $(\mathrm{P} \leqslant \mathbf{0}, 05)$. 


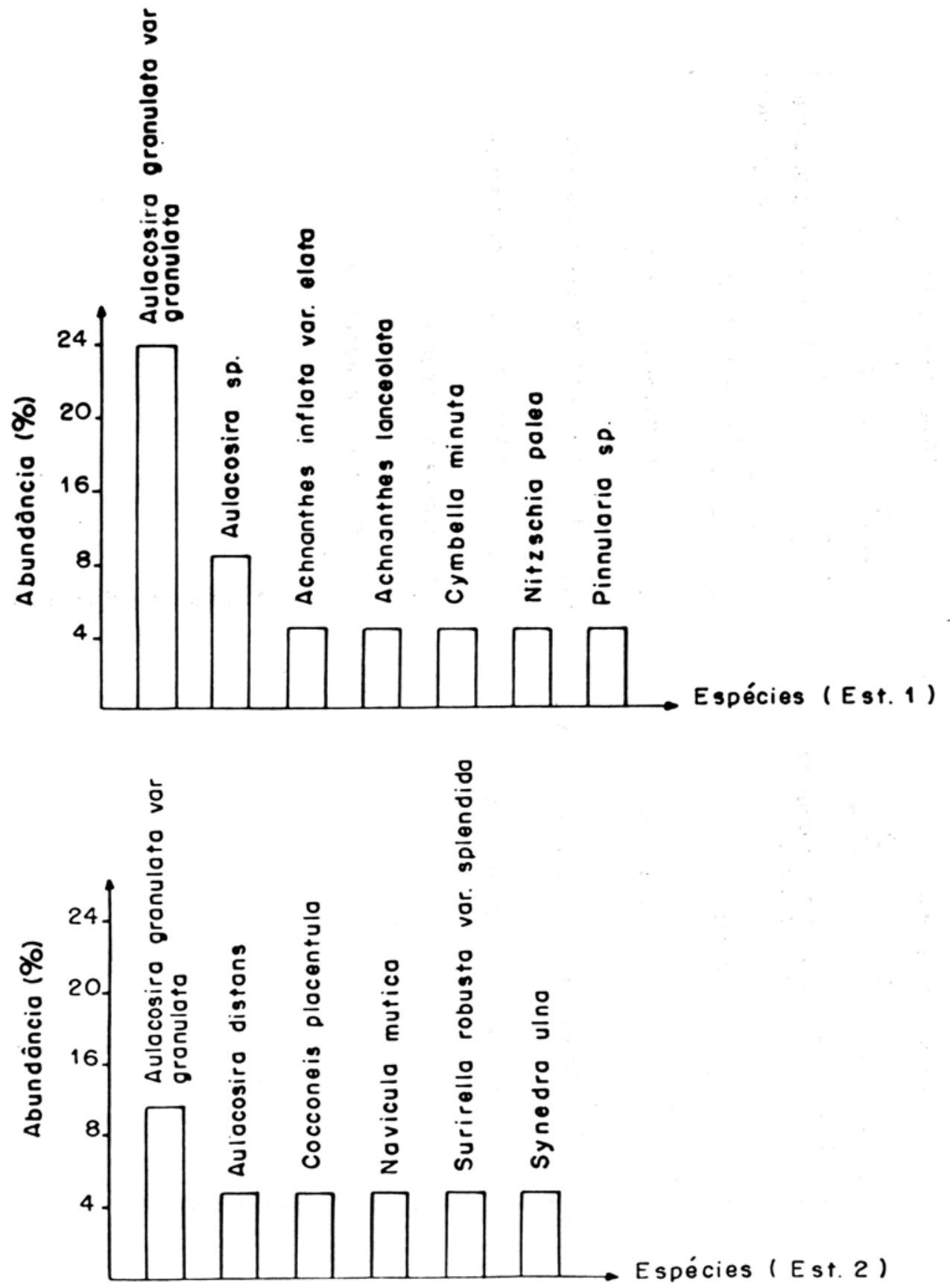

Fig. 3. Espécies abundantes das estaçōes de coleta 1 e 2, em um transecto do sistema Guaíba, no mês d $\epsilon$ setembro de 1975. 

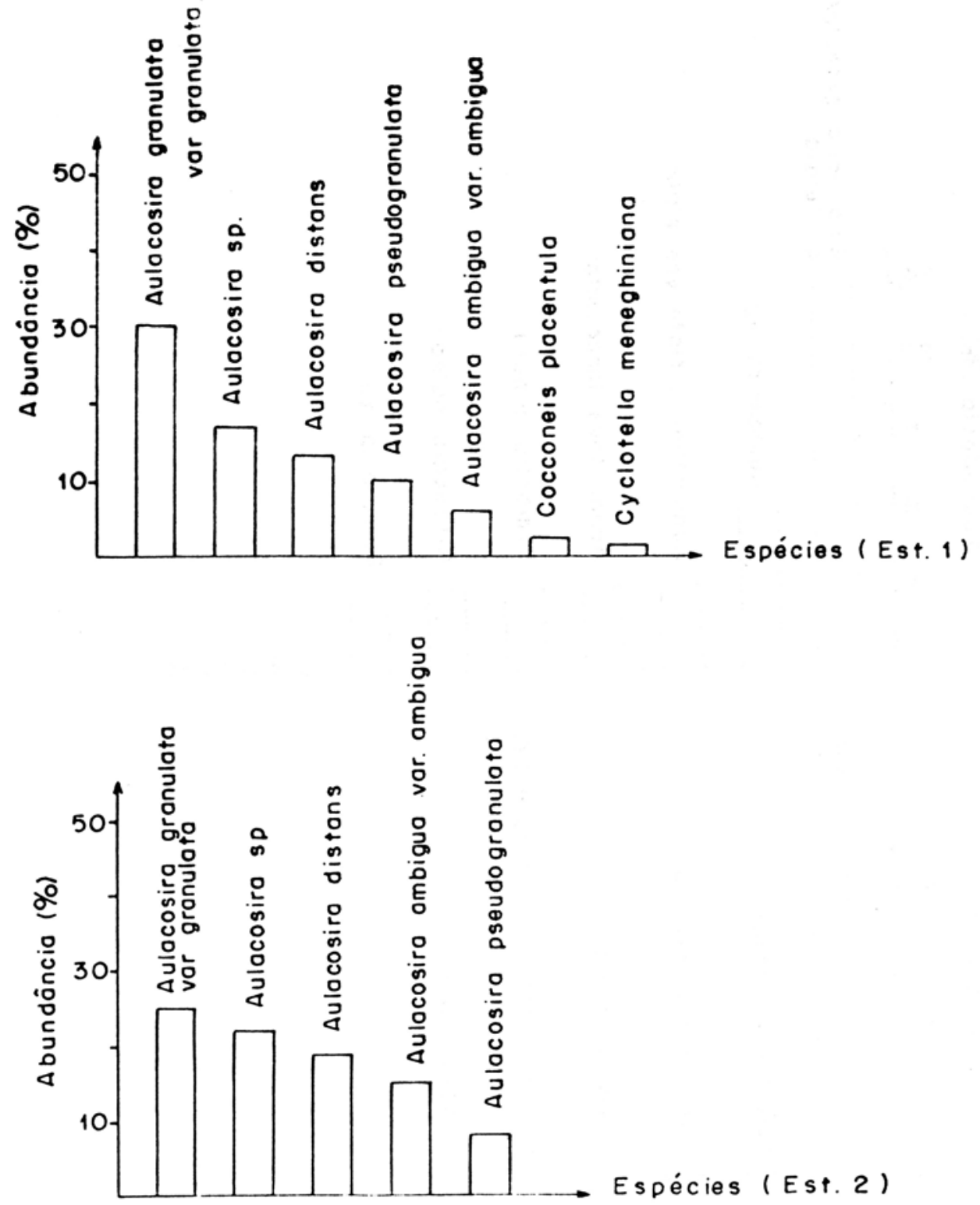

Fig. 4. Espécies abundantes das estaçōes de coleta 1 e 2, em um transecto do sistema Guaf́ba, no mês de novembro de 1975. 


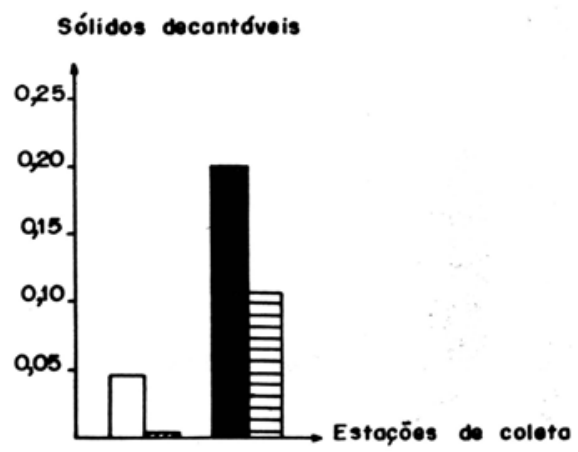

Oxigônio dissolvido

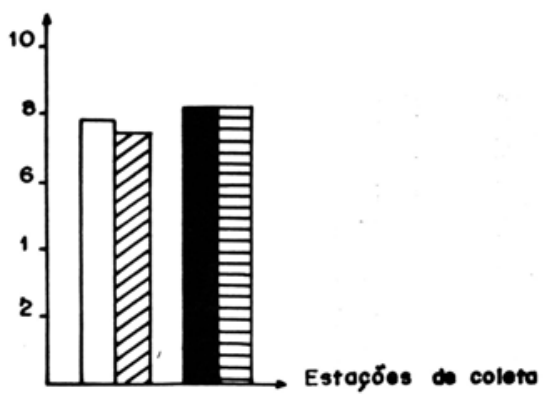

$\square$ Set. 1975 Est. 1

Qet. 1975 Est. 2

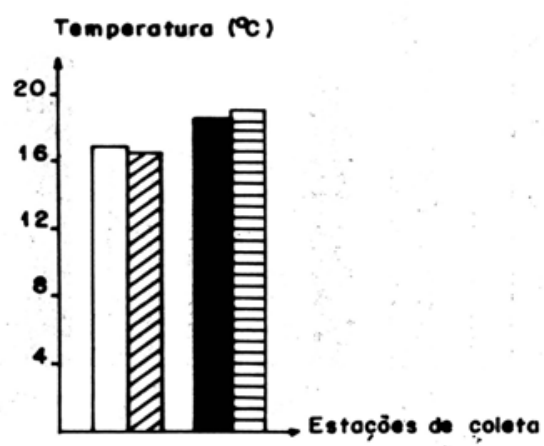

D.B.0. 5 dios (mg./ 1 )

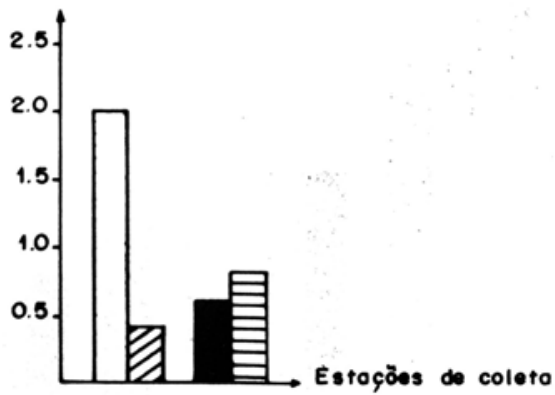

Nov. 1975 Est. 1

Nov. 1975 Est. 2

Fig. 5. Variáveis físicas e químicas que foram medidas nas estaçōes de coleta 1 e 2, do sistema Guarba, durante os meses de setembro e novembro de 1975. 

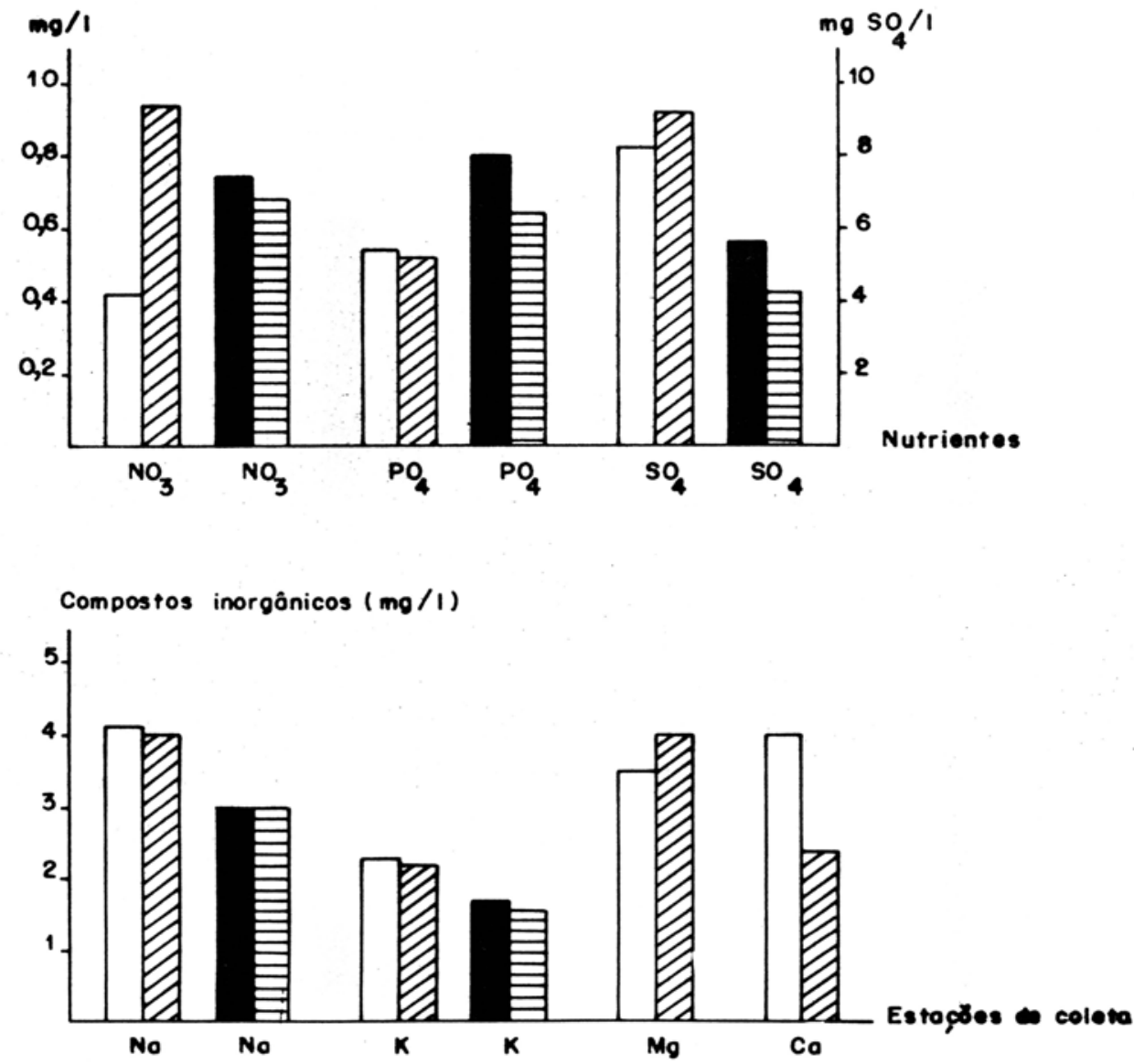

$\square$ Set. 1975 Est. 1

Nov. 1975 Est.1

Set. 1975 Est. 2

Nov. 1975 Est. 2

Fig. 6. Variáveis químicas que foram medidas nas estaçōes de coleta 1 e 2, do sistema Guaíba, durante os meses de setembro e novembro de 1975. 


\section{Conclusões}

Considerando que o estudo foi desenvolvido nas estações 1 e 2 do sistema Guaßba, durante os meses de setembro e novembro de 1975, conclui-se que:

1. No mês de setembro, não houve diferenças significativas entre os índices de diversidade obtidos nas estações 1 e 2. No entanto, foram observadas diferenças na composição especffica de ambas comunidades.

2. Essa diferença na estrutura das comunidades deve-se, provavelmente, à presença exclusiva de Achnanthes inflata var. elata, A. lanceolata, var. lanceolata, Cymbella minuta e Nitzschia palea na estação 1, espécies que foram abundantes e, portanto, características desse ambiente, o qual pode ser definido como tendo níveis B-alfa-mesossapróbios de poluição, com altas concentrações de compostos inorgânicos dissolvidos, principalmente cálcio e potássio, e de Aulacosira distans, Cocconeis placentula, Navicula mutica, Surirella robusta var. splendida e Synedra ulna na estação 2, espécies que foram ábundantes e, portanto, características desse ambiente, o qual pode ser definido como tendo níveis Bmesossapróbicos de poluição, com altas concentrações de nutrientes, principalmente nitrogênio.

3. No mês de novembro, a estação 1 apresentou diversidade da comunidade significativamente maior do que a estação $2(P \leqslant 0,05)$.

4. Essa diferença na estrutura das comunidades deve-se, provavelmente, à presença exclusiva de Cocconeis placentula e Cyclotella meneghiniana na estação 1, visto que o total de táxons abundantes na estação 2 , foi também abundante na estação 1 . Os táxons acima citados são característicos do ambiente da estação 1, o qual pode ser definido como tendo níveis mesossapróbios de poluição, com altas concentraçōes de nutrientes, principalmente fosfato e sulfato.

5. A alta concentração de sólidos decantáveis registrada na estação 1 , tanto em setembro como em novembro, foi uma das principais fontes de poluição proveniente, provavelmente, da descarga de despejos industriais da ex-indústria de celulose Borregaard, cujo vertedouro situa-se próximo à área de amostragem.

\section{Agradecimentos}

Aos técnicos do CESB do Departamento Municipal de Águas e Esgotos de Porto Alegre (DMAE), pelo auxilio na realização das coletas. Ao engenheiro Wilson Chignatti, diretor do DMAE, por autorizar a utilização dos dados f́́sicos e químicos apresentados nesse trabalho. Ao engenheiro Enio Henrique Leite do Departamento do Meio Ambiente, da Secretaria da Saúde e do Meio Ambiente do Estado, pelo apoio dispensado. Às funcionárias Rejane Rosa, Maria Fátima Vieira Machado da. Fundação Zoobotânica do Rio Grande do Sul, pelo apoio técnico prestado:

\section{Referências Bibliográficas}

BILLINGS, R.M. \& DeHAAS, G.G. 1971. Pollution control in the Pulp and Paper Industry. In: LUND, H.F. Industrial pollution control Handbook. MacGraw Hill Book Company, New York, p.1-16.

CLEVE-EULER, A. 1932. Die Kieselalgen des Takernsees in Schweden. K. svenska VetenskAkad. Handl. 11: 3-254.

CLEVE-EULER, A. 1951-1955. Die Diatomeen von Schweden und Finland. K. svensia VetenskAkad. Handl., 2: 1-163, 1951; 3: 1-153. il., 1952; 4: 1-158, il., 1953; 4:1-225, il., 1953; 5: 1-232, il., 1955.

COLLIER, D.B., COX, W.G., JOHNSON, W.A., MILLER, C.P. 1973. Dynamic Ecology. Prentice Hall, Inc. New Jersey.

COMPERE, P. 1982. Taxonomic Revision of the Diatom Genus Pleurosira (Eupodiscaceae). Bacillaria, 5: 165-190.

CONNEL, J.H. \& ORIAS, E. 1964. The ecological regulation of species diversity. American Naturalist, 98: 399-414. 
CÔRTE-REAL, M. \& CALLEGARO, V.L.M. 1973. Catálogo das Bacillariophyceae da costa do Rio Grande do Sul, Brasil. Iheringia, Sér. Bot., Porto Alegre, 17:69-79.

DESCY, J.P. 1979. A new approach to water quality estimation using diatoms. Nova Hedwigia, 64:305-323.

EMILLIANI, M.O.G. de 1973. Ultraestrutura y polimorfismo de Melosira pseudogranulata A. Cl. y cambios en la densidad de su población en el Lago Mascardi. An.Soc.cient.arg.S.Fé, Santa Fé, 1:1-10.

ESTRADA, M. et alii. 1975. Prácticas de ecologia. Universidad Central de Barcelona, Faculdad de Biologia, Departamento de Ecologia, Espanha.

FERREIRA, L.C.H. \& DEWES, R. 1984. Mecânica de correntes do Guaíba. In: SEMINÁRIO SOBRE PESQUISA DA LAGOA DOS PATOS, 1ํ, Porto Alegre, 1984, Súmula, Porto Alegre, Fundação para o Desenvolvimento de Recursos Humanos, p.73-78.

FOX, M.E. 1977. Fate of selected organic compound in the descharge of kraft paper mills into lake Superior. In: KEITH L.H. (ed.) Identification of organic pollutants in waters. Ann Arbor Science Publishers, Michigan, p.300-12.

FRENGUELLI, J. 1942. XVII Contribución al conocimiento de las Diatomeas Argentina. Diatomeas del Neuquén (Patagonia). Revta. Mus. La Plata, 5:73-219.

HENDEY, N.I. 1964. Bacillariophyceae (Diatoms). In: . . An introductory account of the smaller algae of british coastal waters. London, Her Magesty's Stationery Ofice. p.73-158 (Fishery Investigations, Séries 4).

HUBER-PESTALOZZI, G. 1942. Diatomeen. In: . Das Phytoplankton des Susswassers; Systematik und Biologie. Stuttgard, E. Schweizer-bart'sche, p.367-549, il. (Die Binnengewasser, ed. Dr. August Thienemann, 16).

HUSTEDT, F. 1930. Bacillariophyta (Diatomeae). 2. ed. Jena, Gustav Fisher. (Die Sussawasser-Flora Mitteleuropas, ed. A. Pascher, 10).

HOBAYASI, H. \& MAYAMA, S. 1982. Most pollution-tolerant diatoms of severely polluted rivers in the vicinity of Tokyo. Jap. J.P Phycol. 33:188-196.

KREBS, C.J. 1972. Ecology, The experimental analysis of distribution and abundance. Harper \& Row, London.

LANGE-BERTALOT, H. 1979. Pollution tolerance of diatoms as a criterion for water quality estimation. Nova Hedwigia, 64:285-304.

LOBO, E.A. 1984. Modalidades estructurales de las fitocenosis limnicas en los sistemas de desembocadura de rios y esteros de la zona central de Chile (V Region). Valparaiso, 101p. Tese (Tesis para optar al grado de Licenciado en Biologia) Facultad de Medicina, Universidad de Valparaiso, Chile, 1984.

LOWE, R.L. 1974. Environmental requirements and pollution tolerance of freshwater diatoms. Cincinnati, Ohio. Environmental Protection Agency, (Environmental Monitoring Series).

LUCHINI, L. \& VERONA, C.A. 1972. Catálogo de las diatomeas Argentinas. Comisión de Investigaciones Científicas de la Provincia de Buenos Aires.

MARGALEF, R. 1974. Ecologia. Editorial. Editorial Omega, Barcelona.

MARTAU, L.; AGUiAR, L.W. \& CALLEGARO, C.L.M. 1977. Diatomáceas do rio dos Sinos, Rio Grande do Sul, Brasil. Iheringia. Sér. Bot. Porto Alegre (22):45-83.

MULLER-MELCHERS, F.C. \& FERRANDO, H.J. 1956. Técnica para el estudio de las diatomeas. Bolm.Inst.Oceanogr., São Paulo, 7:151-60.

ODUM, E.P. 1972. Ecologia, 3ed. Interamericana. México, 639p.

PATRICK, R. 1971. The effects of increasing light and temperature on the struture of diatom communities. Limnol. Oceanogr., Baltimore, 16(2):405-421.

PATRICK, R. \& REIMER, C.W. 1966. The diatoms of the United State; exclusive of Alaska and Hawai. Philadelphia, Livingsto (Monographs of the Academy of Natural Science of Philadelphia, 13).

PATRICK, R. \& REIMER, C.W. 1975. The diatoms of the United States; exclusive of Alaska and Hawai. Philadelphia, Livingston (Monographs of the Academy of Natural Science of Philadelphia, 13).

PERAGALLO, H. \& PERAGALLO, M. 1897-1908. Diatomées marines de France et des districts maritimes voisins. M.J. Tampère, Grez-sur-Loing.

PIELOU, E.C. 1975. Ecological diversity. Wiley, New York.

PORTO ALEGRE. Prefeitura Municipal. DMAE. CESB. 1971. O rio Gualba, suas características fisicoquímicas e biologicas. Porto Alegre.

PORTO ALEGRE. Prefeitura Municipal. DMAE. CESB. 1974. Ocorrência de superpopulação de organismos planctónicos do rio Guaba e afluentes. Porto Alegre. (DMAE, 11).

PORTO ALEGRE. Prefeitura Municipal. DMAE. CESB. 1976. Análise fisico-químicas e biologicas. Porto Alegre.

PORTO ALEGRE. Prefeitura Municipal. DMAE. CESB. 1978. O rio Guaiba suas caracteristicas ftsicoquimicas e biologicas. Porto Alegre, 15-359p. (DMAE, 27).

PORTO ALEGRE. Prefeitura Municipal. DMAE. CESB. 1981. A medidda da clorofila " $a$ " como índice de produtividade primária no rio Guaba - Porto Alegre. Porto Alegre (DMAE, 32).

SAIZ, F. 1980. Experiencias en el uso de criterios de similitud en el estudio de comunidades. Arch.Biol.Med.Exp., 13:387-402.

SANDERS, H.L. 1968. Marine benthic diversity: a comparative study. American Naturalist, 102:243-82.

SCHOEMAN, F.R. 1976. Diatom indicator groups in the assessment of water quality in the Jukskei-Crocodile River system. (Transvaal, Republic of South Africa).

SCHOEMAN, F.R. 1977. Silica, Nitrogen and Phosphorus requeriments of some Southern Africa Diatoms. J. Limnol. Soc.

SCHOEMAN, F.R. 1979. Diatoms as indicators of water quality in the upper Hennops river (Transvaal, South Africa). J. Limnol.Soc.sth.Afr., 5:73-78. 
SIMONSEN, R. 1979. The diatom system: Ideas on Phylogeny. Bacillaria, 2:9-71.

SLÁDECEK, V. 1973. System of water quality from the biological point of view. Archiv.für Hidrobiologia. p.1-218 (Ergebnisse de limnologie).

STOERMER, E.F.'1963. New taxa and new United States records of the diatom genus Neidium from West Lake Okoboji, Iowa. Notul. Nat., (358):1-9.

TORGAN, L.C. 1978. Diatomáceas do rio Guaíba, Porto Alegre, Rio Grande do Sul, Brasil. Iheringia. Sér. Bot., Porto Alegre, (23):19-63.

TORGAN, L.C. 1979. Segunda nota sobre a flora diatomológica das águas do Guaíba, Porto Alegre, RS. In: SEMANA UNIVERSITARIA GAÚCHA DE DEBATES BIOLÓGICOS, 16. Anais. Porto Alegre, SBRGS, P.43-4 (Resumo).

TORGAN, L.C. 1984. Diatomáceas das praias do Guaíba, Rio Grande do Sul. In. SEMINÁRIO SOBRE PESQUUISA DA LAGOA DOS PATOS, $1^{\circ}$, Porto Alegre, RS. Súmula. Porto Alegre, Fundação para o Desenvolvimento de Recursos Humanos, p.129-33.

TORGAN, L.C. \& AGUIAR, L.W. 1974 Nota preliminar sobre a flora diatomológica do Guaíba, RS. In: CONGRESSO NACIONÁL DE BOTANICA, 25․ Anais. Recife, SBB p.141-2. (Resumo).

VAN HEURCK, H. 1880-1881. Synopsis des diatomées de Belgigue; atlas, Edité par 1'Auteur. Anvers.

VAN LANDINGHAM, S.L. 1964. Some physical and generic aspects of fluctuations in non-marine plankton diatom populations. Bot.Rev., 30:437-79.

WERNER, D. ed. 1977. The biology of diatoms. University of California Press, Berkeley. (Botanical Monographs, 13). 